\title{
The Development of Essential Fatty Acid Deficiency in Healthy Men Fed Fat-Free
}

\section{Diets Intravenously and Orally}

\author{
Jamie D. Wene, William E. Connor, and Lawrence DenBesten \\ From the Clinical Research Center and the Departments of Internal Medicine \\ and Surgery, University of Iowa, College of Medicine, Iowa City, Iowa 52242
}

A B S T RACT The hypothesis that clinical and biochemical essential fatty acid deficiency (EFA) might occur from the feeding of eucaloric, fat-free diets was tested in two experiments in healthy men. In Study I, eight men were given fat-free, eucaloric diets containing $80 \%$ of calories as glucose and $20 \%$ as amino acid hydrolysates by a constant drip over a $24-\mathrm{h}$ period. The diets were fed in succession for periods of $2 \mathrm{wk}$ each, either through a superior vena cava catheter or via a nasogastric tube. EFA deficiency was detected by decreases in linoleic acid and by the appearance of 5,8 , 11-eicosatrienoic acid in lipid fractions of plasma. Linoleic acid decreased significantly during 2 wk of the fat-free diet given intravenously from 48.8 to $9.8 \%$ (percent of total fatty acids) in cholesterol esters, from 21.2 to $3.2 \%$ in phospholipids, from 9.6 to $2.0 \%$ in free fatty acids, and from 14.1 to $2.6 \%$ in triglycerides. Eicosatrienoic acid, normally undetectable, appeared $0.6 \%$ in cholesterol esters, $2.5 \%$ in phospholipids, $0.2 \%$ in free fatty acids, and $2.3 \%$ in triglycerides. EFA deficiency occurred similarly during the nasogastric feeding.

In Study II a subject received the same diet continuously by the nasogastric route for 10 days followed by a 24-h fast. He was then given the fat-free diet intermittently in three meals per day for 3 days. Finally, he was repleted with a diet containing $2.6 \%$ linoleic acid. By the 3rd day of the continuous nasogastric feeding, linoleic acid had fallen significantly and eicosatrienoic acid had appeared in plasma lipid fractions as in Study I. These findings were accentuated by day 10 . Adipose tissue fatty acid composition did not change. Free fatty

\footnotetext{
Presented in part at the 1973 Annual Meeting of the Central Society for Clinical Research.

Received for publication 10 October 1974 and in revised form 19 March 1975.
}

acid outflow from adipose tissue was presumably suppressed during the 10 days of continuous feeding. With increased free fatty acid outflow during fasting and intermittent feeding, linoleic acid rose and eicosatrienoic acid decreased. After 13 days of repletion with dietary linoleic acid, the EFA deficiency had disappeared.

These results indicated that EFA deficiency readily develops when fat-free diets containing glucose are given intravenously or orally as constant 24 -h infusions. These diets are similar to the hyperalimentation formulas now being used clinically.

\section{INTRODUCTION}

Essential fatty acid $(\text { EFA })^{1}$ deficiency was first produced experimentally in young, growing rats by Burr and Burr in 1929 (1). This syndrome has also been described in many other species including man (2). The term "essential fatty acid" refers to linoleic acid (cis, cis-9,12-octadecadienoic acid). Linolenic acid (cis, cis, cis-9,12,15-octadeocatinenoic acid) will stimulate growth but will not relieve the dermal symptoms associated with EFA deficiency, while linoleic acid will effectively correct both (3). Arachidonic acid (cis, cis, cis, cis-5,8,11,14-eicosatetraenoic acid) is approximately three times as effective as linoleic acid in promoting growth (2), but is not an essential nutrient since linoleic acid can be converted to arachidonic acid in the body (4). Linoleic acid then is the EFA, i.e., that fatty acid which must be obtained from the diet by most species to prevent the symptoms of EFA deficiency.

The symptomatology of EFA deficiency falls into three categories: (a) gross abnormalities (e.g., cessation of growth, dermatitis, loss of hair, and increased

\footnotetext{
${ }^{1}$ Abbreviation used in this paper: EFA, essential fatty acid.
} 
TABLE I

Composition of the Hyperalimentation Diet

\begin{tabular}{llc}
\hline $\begin{array}{l}\text { Casein hydrolysate } \\
\text { Dextrose }\end{array}$ & & $20 \%$ \\
Caloric density & & $80 \%$ \\
Electrolytes & Sodium & $1 \mathrm{kcal} / \mathrm{ml}$ \\
& Potassium & $30 \mathrm{meq} /$ liter \\
& Calcium & $15 \mathrm{meq} /$ liter \\
& Chloride & $12.5 \mathrm{meq} / \mathrm{liter}$ \\
& Magnesium & $19.6 \mathrm{meq} / \mathrm{liter}$ \\
& Phosphorus & $4.2 \mathrm{meq} / \mathrm{liter}$ \\
& Thiamine hydrochloride & $24.6 \mathrm{meq} / \mathrm{liter}$ \\
Vitamins & Riboflavin & $10 \mathrm{mg}$ \\
& Sodium pantothenate & $20 \mathrm{mg}$ \\
& Nicatinamide & $100 \mathrm{mg}$ \\
& Pyridoxine hydrochloride & $20 \mathrm{mg}$ \\
& Ascorbic acid & $500 \mathrm{mg}$ \\
\hline
\end{tabular}

susceptibility to bacterial infections), (b) histological abnormalities (e.g., microscopic changes in the kidneys, skin, ovaries, and testes), and (c) physiological and biochemical abnormalities $(2,5)$. The last category includes biochemical changes in the fatty acid patterns of blood and organs such as liver and heart (6). These changes involve increased levels of $5,8,11$-eicosatrienoic acid and decreased levels of linoleic acid and arachidonic acid. The changes in blood levels may be the earliest signs of EFA deficiency (2).

Experimental studies have shown that the very young animals are much more susceptible to EFA deficiency than adults (7-9). Growing animals require linoleic acid and their body stores are low, thereby making depletion easily producible. Because the dietary requirement is low and the storage of linoleic acid in adipose tissue is great, it has been extremely difficult to produce clinical EFA deficiency in adults. Most of the human studies reported to date have been in infants and children (10-14). Only two cases of linoleic acid deficiency have been reported in adult humans. Collins et al. found a deficiency secondary to short bowel syndrome in one adult patient and a deficiency resulting from a colectomy with severe malnutrition in another (15). Up to this time, EFA deficiency has not been reported in healthy adult humans.

An understanding of EFA deficiency has an immediate importance for present-day medicine because the sole source of nutrition for many severely ill patients is intravenous hyperalimentation. The infusate is fatfree, consisting of amino acid hydrolysates, glucose, minerals, and vitamins. The diet is, therefore, EFA deficient. In chronically ill, malnourished individuals it is not known whether the lack of dietary linoleic acid might have deleterious effects. The studies reported here were designed to ascertain if such a diet would cause EFA deficiency in adult humans.

\section{METHODS}

Study I. Eight healthy male volunteers ${ }^{2}$ from the Iowa State Penitentiary were admitted to the Clinical Research Center. A hyperalimentation diet was fed both intravenously and nasogastrically (Table I). This diet, "Central Venous Nutrient Solution," was $80 \%$ glucose, $20 \%$ casein amino acid hydrolysates (Amigen ${ }^{\circledR}$, Baxter Laboratories, Morton Grove, Ill.) and was completely fat free. Appropriate electrolytes and vitamins were added to meet the daily nutritional requirements. Sterile solutions were prepared daily at a caloric equivalent of $1 \mathrm{kcal} / \mathrm{ml}$. To minimize the effect of weight change on serum lipids, a careful dietary history was taken on admission to the Clinical Research Center, and caloric needs were estimated both from this information and from the height and weight. The men were ambulatory throughout the study and engaged in the usual ward recreational activities.

During the base-line 7-day period of the study, all subjects were fed a eucaloric general diet. The linoleic acid content of this diet was $2-3 \%$ of total calories. During the next two 14-day sequential periods each subject was fed the eucaloric, fat-free hyperalimentation diet either by nasogastric tube or intravenous catheter. The solutions were infused at a constant rate over $24 \mathrm{~h}$. Four subjects in Group A received this nutrient solution for 14 days intravenously and then nasogastrically for another 14 days. The four subjects in Group B received this solution in reverse order. Each subject, therefore, was given the fat-free diet for a total of 28 days.

The formula was infused through a 17-gauge plastic catheter which had been threaded through the subclavian vein into the superior vena cava under fluoroscopic control. All infusing solutions were passed through a $44 \mu \mathrm{m}$ filter placed immediately proximal to the subclavian catheter. Every 3rd day all dressings were removed and skin around the catheter was defatted with acetone. All catheter tips were cultured at the termination of the study and found to be sterile.

Study II. Subject E. R., a healthy male volunteer 21 yr of age, was admitted to the Clinical Research Center for a more detailed study of the changes in serum fatty acids which occurred in Study I subjects. A eucaloric formula diet containing $2.6 \%$ of calories as linoleic acid was first fed for $2 \mathrm{mo}$. This was followed by four dietary periods: In Period A, a completely fat-free diet was administered by constant nasogastric drip for 10 days. Period B was a 24-h fast. In Period C, the fat-free diet was divided into three equal meals and was fed intermittently via nasogastric tube at 8 a.m., 12 noon, and 4 p.m. for 3 days. In Period D, a 2-wk repletion period, a formula diet containing $2.6 \%$ of calories as linoleic acid was fed.

Fatty acid analysis. The lipids of the serum were extracted with chloroform-methanol $(2: 1)$ (16). Separation of lipid classes was carried out by preparative thin-layer chromatography on Silica Gel G using hexane-ether-chloroform-acetic acid (80:10:10:1 vol/vol) as the developing solvent (17). The plates were visualized using Rhodamine 6G (Matheson, Coleman, and Bell, Norwood, Ohio) and ultraviolet light, and the bands of cholesterol esters, tri-

2 The proposed study was fully explained to each man and informed, written consent was obtained. The protocol of the study had been approved by the College of Medicine Research Committee in charge of all prison volunteer studies and by the Human Use Committee. The study was in accord with the principles of the Declaration of Helsinki. 
TABLE II

Study I-The Changes in the Fatty Acid Composition of Plasma of Eight Subjects after a Fat-Free_Diet*

\begin{tabular}{|c|c|c|c|c|c|c|c|c|c|c|}
\hline & \multicolumn{10}{|c|}{ Fatty acids expressed as percent of total fatty acids (mean $\pm \mathrm{SD}$ ) } \\
\hline & \multicolumn{5}{|c|}{ Group A (IV first 14 days; NG second 14 days) } & \multicolumn{5}{|c|}{ Group B (NG first 14 days; IV second 14 days) } \\
\hline & & $\mathrm{CE}$ & PPL & FFA & TG‡ & & $\mathrm{CE}$ & PPL & FFA & TG \\
\hline \multicolumn{11}{|c|}{ Palmitic acid C16:0 } \\
\hline Base line & & $15.1 \pm 4.1$ & $26.6 \pm 2.3$ & $27.4 \pm 7.2$ & $31.3 \pm 5.0$ & & $14.5 \pm 2.8$ & $27.8 \pm 2.5$ & $29.0 \pm 2.4$ & $29.9 \pm 6.8$ \\
\hline 14 days & IV $\ddagger$ & $14.1 \pm 0.3$ & $30.7 \pm 2.0$ & $34.9 \pm 1.2$ & $42.3 \pm 10.7$ & NG & $17.0 \pm 0.8$ & $33.9 \pm 2.6$ & $35.4 \pm 5.38$ & $40.2 \pm 2.2 \|$ \\
\hline 28 days & NG & $20.5 \pm 12.6$ & $29.3 \pm 2.2$ & $30.9 \pm 3.1$ & $38.5 \pm 2.6$ & IV & $14.2 \pm 1.7$ & $31.3 \pm 3.4$ & $33.4 \pm 4.3 \S$ & $36.7 \pm 2.2$ \\
\hline \multicolumn{11}{|c|}{ Palmitoleic acid $\mathrm{C} 16: 1$} \\
\hline Base line & & $3.5 \pm 0.8$ & $1.0 \pm 0.3$ & $7.3 \pm 4.8$ & $1.8 \pm 0.4$ & & $5.6 \pm 1.3$ & $1.1 \pm 0.3$ & $5.9 \pm 0.6$ & $1.9 \pm 0.8$ \\
\hline 14 days & IV & $19.5 \pm 3.5$ & $2.5 \pm 0.5 \S$ & $9.7 \pm 1.4$ & $3.5 \pm 1.6$ & NG & $18.1 \pm 3.2 \|$ & $2.3 \pm 0.7 \S$ & $8.9 \pm 2.6$ & $3.8 \pm 1.1$ \\
\hline 28 days & NG & $17.6 \pm 7.2^{* * *}$ & $2.5 \pm 1.2$ & $9.9 \pm 3.2$ & $3.6 \pm 1.3 \S$ & IV & $18.9 \pm 5.1 \|$ & $2.2 \pm 0.3 \|$ & $8.0 \pm 4.0$ & $5.1 \pm 3.2$ \\
\hline \multicolumn{11}{|c|}{ Stearic acid C18:0 } \\
\hline Base line & & $1.7 \pm 0.2$ & $18.4 \pm 1.4$ & $3.9 \pm 0.4$ & $18.4 \pm 2.2$ & & $1.5 \pm 0.5$ & $18.5 \pm 1.5$ & $4.0 \pm 0.2$ & $21.4 \pm 1.7$ \\
\hline 14 days & IV & $0.9 \pm 0.1 \Upsilon$ & $17.9 \pm 1.2$ & $4.2 \pm 0.9$ & $18.4 \pm 3.7$ & NG & $0.9 \pm 0.1 \|$ & $14.9 \pm 0.8 \S$ & $5.1 \pm 1.1$ & $13.2 \pm 1.5 \S$ \\
\hline 28 days & NG & $1.1 \pm 0.4 \Upsilon$ & $17.0 \pm 2.8$ & $3.5 \pm 0.7$ & $15.5 \pm 4.4$ & IV & $0.7 \pm 0.2 \|$ & $16.7 \pm 1.7$ & $6.0 \pm 5.4$ & $12.8 \pm 6.5$ \\
\hline \multicolumn{11}{|c|}{ Oleic acid C18:1 } \\
\hline Base line & & $26.8 \pm 2.0$ & $18.4 \pm 2.9$ & $47.9 \pm 9.5$ & $25.4 \pm 6.0$ & & $29.9 \pm 2.1$ & $17.3 \pm 1.5$ & $45.2 \pm 3.0$ & $20.9 \pm 2.8 \S$ \\
\hline 14 days & IV & $48.7 \pm 4.0$ ฯ & $30.9 \pm 1.99$ & $45.7 \pm 2.3$ & $22.1 \pm 12.2$ & NG & $44.6 \pm 5.7 \|$ & $24.0 \pm 2.3 \|$ & $43.8 \pm 7.0$ & $25.5 \pm 0.8$ \\
\hline 28 days & NG & $42.9 \pm 3.9 ף$ & $29.0 \pm 4.8 \|$ & $48.1 \pm 4.5$ & $29.7 \pm 2.2$ & IV & $41.6 \pm 4.1 \|$ & $24.3 \pm 2.6 * *$ & $41.1 \pm 8.0$ & $30.3 \pm 8.7$ \\
\hline \multicolumn{11}{|c|}{ Linoleic acid C18:2 } \\
\hline Base line & & $48.8 \pm 2.1$ & $21.2 \pm 2.8$ & $9.6 \pm 5.5$ & $14.1 \pm 2.8$ & & $40.2 \pm 3.7$ & $16.6 \pm 2.4$ & $10.1 \pm 3.0$ & $14.5 \pm 3.2$ \\
\hline 14 days & IV & $9.8 \pm 3.6$ & $3.2 \pm 1.3 \ddagger \ddagger$ & $2.0 \pm 1.0 \S$ & $2.6 \pm 1.8 \Phi$ & NG & $13.5 \pm 4.2 \|$ & $6.6 \pm 2.7 \|$ & $2.7 \pm 1.7 \Phi$ & $5.0 \pm 1.7$ \\
\hline 28 days & NG & $12.7 \pm 11.4^{* *}$ & $4.3 \pm 2.2 \ddagger \ddagger$ & $2.8 \pm 2.7$ & $3.1 \pm 2.79$ & IV & $11.6 \pm 8.7 \|$ & $5.3 \pm 2.1^{* *}$ & $3.8 \pm 1.4 \|$ & $4.1 \pm 2.9 * *$ \\
\hline \multicolumn{11}{|c|}{ Eicosatrienoic acid C20:3 $3 \omega 9$} \\
\hline Base line & & 0 & 0 & $0.1 \pm 0.1$ & 0 & & 0 & 0 & 0 & 0 \\
\hline 14 days & IV & $0.6 \pm 0.2 * *$ & $2.5 \pm 1.3 \S$ & $0.2 \pm 0.1$ & $2.3 \pm 1.0 \|$ & NG & $0.4 \pm 0.2 \S$ & $2.6 \pm 0.8^{* *}$ & $0.2 \pm 0.1 \|$ & $1.9 \pm 1.2 \S$ \\
\hline 28 days & NG & $0.9 \pm 0.2 \rrbracket$ & $3.5 \pm 1.6 \|$ & $0.3 \pm 0.1 \S$ & $2.5 \pm 1.7$ & IV & $1.3 \pm 0.5 \|$ & $4.2 \pm 1.7 \|$ & $1.6 \pm 1.3$ & $2.4 \pm 2.2 \|$ \\
\hline \multicolumn{11}{|c|}{ Arachidonic acid C20:4 } \\
\hline Base line & & $3.7 \pm 1.2$ & $9.3 \pm 2.0$ & $0.5 \pm 0.4$ & $6.5 \pm 3.8$ & & $5.1 \pm 2.4$ & $11.3 \pm 0.7$ & $1.5 \pm 0.9$ & $8.2 \pm 3.9$ \\
\hline 14 days & IV & $3.8 \pm 1.8$ & $7.4 \pm 2.0$ & $0.7 \pm 0.6$ & $5.5 \pm 1.9$ & NG & $3.0 \pm 2.0$ & $8.3 \pm 2.5 \S$ & $0.6 \pm 0.1$ & $5.2 \pm 1.2$ \\
\hline 28 days & NG & $2.7 \pm 2.0$ & $8.1 \pm 2.2$ & $0.4 \pm 0.3$ & $3.7 \pm 2.2$ & IV & $5.6 \pm 1.0$ & $10.0 \pm 1.9$ & $3.2 \pm 2.6$ & $4.8 \pm 2.3$ \\
\hline
\end{tabular}

* Minor fatty acids not included: C14:0, C15:0, C17:0, C18:3, C20:0, C20:3w6.

$\ddagger$ CE, cholesterol esters; PPL, phospholipids; FFA, free fatty acids; TG, triglycerides; IV, intravenous feeding; NG, nasogastric feeding.

$\S$ Change from base line significant according to paired $t$ test, $P<0.05$.

$\|$ Change from base line significant according to paired $t$ test, $P<0.025$.

I Change from base line significant according to paired $t$ test, $P<0.005$.

** Change from base line significant according to paired $t$ test, $P<0.01$.

$\ddagger$ Change from base line significant according to paired $t$ test, $P<0.001$.

glycerides, and free fatty acids were collected. The rest of the plate was developed in diethyl ether to remove impurities from the phospholipid band which was then collected. The fatty acid composition of the isolated cholesterol esters, free fatty acids, phospholipids, and triglycerides was determined by gas-liquid chromatography from methylesters prepared with boron fluoride methanol (18). The gas-liquid chromatograph used was a Hewlett-Packard $7610 \mathrm{~A}$ instrument with a flame ionization detector and 6foot dual columns (Hewlett-Packard Co., Palo Alto, Calif.). The carrier gas was helium and the stationary phase was $6 \%$ diethylene glycol succinate on $80 / 100$ Diatoport S. The samples were run at $135-200^{\circ} \mathrm{C}$ with a $2 \mathrm{~min}$ programmed temperature rise. The helium flow was $50 \mathrm{ml} / \mathrm{min}$. A standard prepared from a mixture of Hormel Institute GLC standards was run at the beginning of each day, and sample peaks were compared to those of the standards by the use of relative retention times for identification. Fatty acid peaks were quantitated on a weight percentage basis. Data in Study $I$ is expressed as the mean \pm the SD for one sample in each subject at the indicated day. The linoleic acid content of the formula diet in Study II was likewise determined by analysis of an aliquot. Statistical comparisons of paired data were made by Student's $t$ test (19). The overall coefficient of variation for the combined thin-layer and gas-liquid chromatography determinations of all fatty acids was $3.52 \%$. For large peaks such as linoleic and oleic acids, the coefficient of variations was $1.0 \%$ or less.

\section{RESULTS}

Study I. During the base-line dietary period, the eight men in Study I had serum fatty acid patterns similar to results reported by others for normals as indicated in Table II $(20,21)$. All subjects developed biochemical EFA deficiency within 2 wk after begining the fat-free diet. Mean linoleic acid levels fell and eicosatrienoic acid levels rose in all serum lipid frac- 
TABLE III

Study II-The Changes in the Fatty Acid Composition of

\begin{tabular}{|c|c|c|c|c|c|c|c|c|c|c|c|c|c|c|}
\hline & \multicolumn{7}{|c|}{ Cholesterol esters } & \multicolumn{7}{|c|}{ Phospholipids } \\
\hline & $\mathrm{C} 16: 0$ & C16: 1 & C18:0 & C18: 1 & C18:2 & $\begin{array}{c}\text { C20: } \\
3 \omega 9\end{array}$ & $\mathrm{C} 20: 4$ & C16:0 & C16: 1 & C18:0 & C18: 1 & C18:2 & $\begin{array}{c}\text { C20: } \\
3 \omega 9\end{array}$ & $\mathrm{C} 20: 4$ \\
\hline Base line & 10.2 & 1.8 & 1.9 & 35.5 & 45.6 & 0 & 3.0 & 25.6 & 0.5 & 17.0 & 19.6 & 23.0 & $\operatorname{tr}$ & 8.5 \\
\hline \multicolumn{15}{|c|}{ Period A-fat-free } \\
\hline \multirow{6}{*}{ Day } & 10.9 & 2.1 & 1.8 & 36.5 & 42.9 & 0 & 3.6 & 27.3 & 1.1 & 16.1 & 20.1 & 17.2 & 0.4 & 11.1 \\
\hline & 13.2 & 8.4 & 1.8 & 39.7 & 26.4 & 0 & 5.3 & 27.6 & 3.3 & 15.4 & 25.2 & 6.7 & 0.6 & 13.0 \\
\hline & 15.6 & 14.9 & 1.5 & 46.1 & 14.5 & 0 & 3.0 & 27.4 & 2.7 & 18.1 & 21.9 & 5.6 & 0.9 & 11.4 \\
\hline & 14.9 & 16.8 & 1.6 & 44.9 & 11.8 & 0.2 & 5.3 & 27.6 & 2.8 & 18.0 & 26.9 & 5.6 & 0.9 & 11.1 \\
\hline & 15.0 & 19.0 & 1.3 & 48.3 & 8.8 & 0.5 & 4.0 & 27.8 & 2.7 & 19.0 & 30.9 & 2.6 & 2.6 & 9.2 \\
\hline & 13.7 & 16.9 & 1.3 & 48.6 & 9.4 & 0.8 & 6.0 & 26.8 & 3.0 & 17.4 & 29.3 & 2.8 & 2.7 & 12.8 \\
\hline \multicolumn{15}{|c|}{ Period B-total fast } \\
\hline $0 \mathrm{~h}$ & 13.7 & 16.9 & 1.3 & 48.6 & 9.4 & 0.8 & 6.0 & 26.8 & 3.0 & 17.4 & 29.3 & 2.8 & 2.7 & 12.8 \\
\hline $12 \mathrm{~h}$ & 12.5 & 16.3 & 1.2 & 47.7 & 10.3 & 1.0 & 6.9 & 26.6 & 2.3 & 16.5 & 26.8 & 5.8 & 3.0 & 12.9 \\
\hline $18 \mathrm{~h}$ & 12.6 & 16.3 & 1.1 & 47.7 & 11.9 & 0.8 & 6.1 & 25.9 & 1.4 & 17.3 & 27.0 & 8.2 & 2.7 & 11.0 \\
\hline $24 \mathrm{~h}$ & 12.8 & 16.2 & 1.0 & 45.3 & 13.9 & 0.9 & 6.7 & 26.9 & 1.9 & 14.7 & 23.5 & 10.3 & 3.1 & 13.3 \\
\hline \multicolumn{15}{|c|}{ Period C-intermittent feeding } \\
\hline \multirow{2}{*}{ Day } & 14.2 & 11.0 & 0 & 39.1 & 21.9 & 1.0 & 8.6 & 30.4 & 2.0 & 15.0 & 20.1 & 10.2 & 2.3 & 13.0 \\
\hline & 13.4 & 9.2 & 1.2 & 39.6 & 26.0 & 0.7 & 6.3 & 28.2 & 0.8 & 13.9 & 22.8 & 13.8 & 2.6 & 11.9 \\
\hline \multicolumn{15}{|c|}{ Period D-repletion } \\
\hline \multirow{4}{*}{ Day } & 13.2 & 7.7 & 1.3 & 39.7 & 31.0 & 0.5 & 3.8 & 23.9 & 0.1 & 19.5 & 22.8 & 17.0 & 1.4 & 9.7 \\
\hline & 10.5 & 4.6 & 1.2 & 33.4 & 39.5 & 0.6 & 6.5 & 25.4 & 0.8 & 16.6 & 18.6 & 18.2 & 1.6 & 11.5 \\
\hline & 9.4 & 2.8 & 1.4 & 32.2 & 43.6 & 0.5 & 6.4 & 26.0 & 0.5 & 17.0 & 18.2 & 19.4 & 0.9 & 11.1 \\
\hline & 9.6 & 1.8 & 1.6 & 30.7 & 49.2 & $\operatorname{tr}$ & 5.0 & 26.1 & 0.5 & 16.1 & 18.0 & 23.8 & 0.3 & 10.1 \\
\hline
\end{tabular}

* Fatty acids expressed as percent of total fatty acids.

tions (cholesterol esters, phospholipids, free fatty acids, and triglycerides). An example of this is shown in Fig. 1 which represents the cholesterol ester linoleic and eicosatrienoic acid changes for two of the subjects in Study I. Table II contains the detailed data for all subjects divided into Groups A and B according to the sequence of administration of the diet.

The subjects in Group A received the fat-free formula by intravenous catheter for 14 days immediately

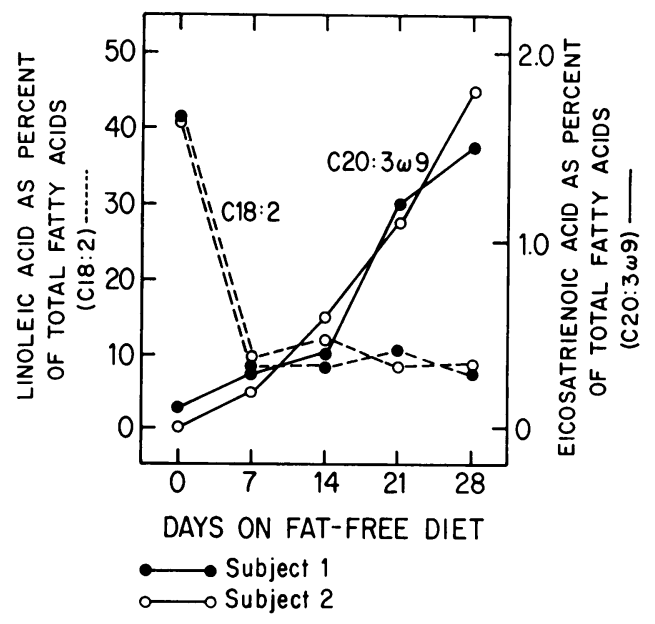

FIgUre 1 The changes in linoleic acid and eicosatrienoic acid as percent of the total fatty acid composition of cholesterol esters in two subjects of Study I. after the base-line period. The first remarkable change in the fatty acid composition was the profound lowering of linoleic acid in each lipid class (Table II). Linoleic acid decreased from 48.8 to $9.8 \%$ in the cholesterol ester fraction, from 21.2 to $3.2 \%$ in the phospholipid fraction, from 9.6 to $2.0 \%$ in the free fatty acid fraction, and from 14.1 to $2.6 \%$ in the triglyceride fraction during the 14 days of the fat-free diet. These differences were all statistically significant $(P<$ 0.005 ).

During the second 14-day period in which Group A received the diet by nasogastric feeding, the mean linoleic acid levels did not change significantly from the values at the end of the intravenous feeding.

The second change of interest seen in the serum fatty acid pattern was the appearance of eicosatrienoic acid in each lipid fraction. During the intravenous feeding, it increased significantly $(P<0.05)$ in each fraction from a base line of zero or trace amounts to $0.6 \%$ in cholesterol esters, $2.5 \%$ in phospholipids, $0.2 \%$ in free fatty acids, and $2.3 \%$ in triglycerides. Levels of eicosatrienoic acid rose still higher after 28 days on a fat-free diet, the last 2 wk of which was administered nasogastrically. These values were not statistically different, however, from those after the 14 days of intravenous fat-free feeding.

Although there were reciprocal changes in linoleic acid and eicosatrienoic acid in all of the lipid classes, 
Plasma of a Single Subject (E. R.) after a Fat-Free Diet*

\begin{tabular}{|c|c|c|c|c|c|c|c|c|c|c|c|c|c|}
\hline \multicolumn{7}{|c|}{ Free fatty acids } & \multicolumn{7}{|c|}{ Triglycerides } \\
\hline $\mathrm{C} 16: 0$ & C16: 1 & C18:0 & C18:1 & $\mathrm{C} 18: 2$ & $\begin{array}{l}\text { C20: } \\
3 \omega 9\end{array}$ & $\mathrm{C} 20: 4$ & $\mathrm{C} 16: 0$ & $\mathrm{C} 16: 1$ & C18:0 & C18: 1 & $\mathrm{C} 18: 2$ & $\begin{array}{c}\text { C20: } \\
3 \omega 9\end{array}$ & C20: 4 \\
\hline 26.1 & 1.8 & 8.6 & 52.5 & 9.0 & 0 & 1.1 & 27.5 & 0.2 & 17.0 & 22.5 & 22.5 & 0 & 6.6 \\
\hline 28.9 & 4.4 & 3.0 & 47.3 & 11.5 & 0 & 1.8 & 28.8 & 1.3 & 17.0 & 21.2 & 15.5 & 0.3 & 10.1 \\
\hline 31.2 & 11.9 & 1.9 & 42.1 & 5.6 & 0.3 & 2.6 & 29.0 & 4.2 & 14.5 & 27.1 & 7.0 & 1.2 & 9.1 \\
\hline 34.6 & 10.6 & 3.2 & 41.8 & 4.2 & 0.3 & 1.9 & 28.8 & 4.2 & 14.2 & 27.4 & 5.9 & 1.4 & 11.4 \\
\hline 34.6 & 12.1 & 2.9 & 43.8 & 2.4 & 0.3 & 1.5 & 29.9 & 5.8 & 14.1 & 30.8 & 3.7 & 1.6 & 9.1 \\
\hline 35.1 & 11.0 & 4.1 & 44.2 & 1.8 & 0.4 & 1.0 & 31.4 & 4.8 & 14.7 & 30.7 & 2.8 & 2.9 & 8.0 \\
\hline 34.5 & 10.7 & 3.9 & 46.3 & 1.8 & 0.4 & 1.1 & 28.1 & 4.3 & 15.0 & 32.5 & 2.9 & 2.9 & 10.3 \\
\hline 34.5 & 10.7 & 3.9 & 46.3 & 1.8 & 0.4 & 1.1 & 28.1 & 4.3 & 15.0 & 32.5 & 2.9 & 2.9 & 10.3 \\
\hline 29.1 & 8.7 & 2.4 & 49.7 & 5.5 & 0.5 & 0.9 & 27.5 & 3.8 & 14.0 & 30.9 & 6.4 & 3.0 & 9.6 \\
\hline 28.0 & 7.1 & 2.4 & 49.7 & 6.7 & 0.6 & 1.9 & 35.8 & 5.3 & 13.0 & 37.5 & - & - & - \\
\hline 28.4 & 6.2 & 2.7 & 50.6 & 7.6 & 0.9 & 1.4 & 31.2 & 3.2 & 10.6 & 27.0 & 10.3 & 3.5 & 9.2 \\
\hline 34.8 & 8.6 & 3.0 & 41.4 & 6.3 & 0.4 & 1.3 & 38.4 & 1.8 & 14.3 & 20.9 & 8.4 & 1.3 & 10.7 \\
\hline 27.7 & 5.4 & 6.9 & 49.5 & 6.0 & 0.3 & 1.2 & 27.2 & 2.4 & 15.6 & 27.9 & 12.0 & 1.6 & 7.7 \\
\hline 27.5 & 4.5 & 7.4 & 50.6 & 6.2 & 0.3 & 1.0 & 26.1 & 1.7 & 16.9 & 26.1 & 14.1 & 1.7 & 8.0 \\
\hline 27.3 & 3.5 & 5.6 & 51.8 & 8.2 & 0.4 & 0.9 & 29.4 & 1.2 & 15.3 & 22.1 & 17.0 & 1.2 & 8.3 \\
\hline 26.9 & 2.8 & 5.7 & 51.6 & 9.9 & 0.2 & 1.1 & 25.3 & 0.9 & 13.7 & 21.9 & 19.2 & 1.5 & 11.1 \\
\hline 29.8 & 3.2 & 5.3 & 49.6 & 12.4 & 0 & 0.9 & 28.1 & 0.7 & 15.4 & 20.2 & 22.8 & 0.3 & 8.4 \\
\hline
\end{tabular}

the percent increases seen in eicosatrienoic acid were not enough to make up for the great decrease in linoleic acid. The monounsaturated fatty acids, palmitoleic (C16:1) and oleic (C18:1), increased to make up this difference (Table I). Arachidonic acid levels decreased significantly only in the phospholipid fraction during the first 14 days of intravenous feeding $(P<$ $0.05)$. Dermatitis, which is usually the first clinical sign of EFA deficiency was not detected at any time in the study.

The subjects in Group B received the fat-free diet for 14 days by constant drip nasogastric feeding immediately after the base-line period. During this period, linoleic acid declined significantly $(P<0.025)$ in all lipid classes (Table II). The greatest drop relative to base line was in the free fatty acid fraction where it decreased from 10.1 to $2.7 \%$ of total fatty acids. Linoleic acid in the cholesterol ester fraction fell from 40.2 to $13.5 \%$, in phospholipids from 16.6 to $6.6 \%$, and in triglycerides from 14.5 to $5.0 \%$. The subsequent intravenous feeding sustained but did not alter significantly the changes which had already ocurred during the first 14 days.

After 14 days of nasogastric fat-free feeding, all subjects of Group B had significantly increased $(P<$ $0.025)$ levels of eicosatrienoic acid: $2.6 \%$ in phospholipid fatty acids, $1.9 \%$ in triglyceride fatty acids, $0.4 \%$ in cholesterol fatty acids, and $0.2 \%$ in free fatty acids. After 28 days on the fat-free diet, 14 days of nasogas- tric feeding followed by 14 days of intravenous feeding, eicosatrienoic acid levels rose significantly $(P<0.05)$ in each fraction above the values seen after the 14 day fat-free nasogastric period.

The percentages of palmitoleic acid and oleic acid again increased to counterbalance the great decrease seen in linoleic acid. For example, in the cholesterol ester fraction, palmitoleic rose from 5.6 to $18.9 \%$ and oleic from 29.9 to $44.6 \%$ during the first 14 days of nasogastric feeding. Arachidonic acid levels decreased significantly $(P<0.05)$ from 11.3 to $8.3 \%$ in the phospholipid fraction during the first 14 days of nasogastric feeding, but not during the second 14 days of intravenous feeding.

Study II (subject E. R.). This second study was undertaken in Subject E. R. to obtain more detailed data of the metabolic processes which occurred during Study I. During four dietary periods, he received in sequence a continuously fed fat-free formula, a 24-h fast, an intermittently fed fat-free formula, and a repletion formula. Linoleic acid decreased markedly in all plasma lipid fractions by the 3rd day of the fat-free diet of Period A (Table III). At this time the amount of linoleic acid in the cholesterol ester and free fatty acid fractions was about half of base line and in phospholipid and triglyceride about one-third of base line. All fractions appeared to have leveled off after days 9 and 10 of the fat-free formula. 
TABLE IV

Study II (subject E. R.)-The Percent Fatty Acid Composition of Adipose Tissue

before and after a Fat-Free Diet

\begin{tabular}{|c|c|c|c|c|c|c|c|c|c|c|}
\hline & C12:0 & C14:0 & C16:0 & C16:1 & C18:0 & C18:1 & C18:2 & C20:0 & $\begin{array}{c}\text { C20: } \\
3 \omega 9\end{array}$ & C20:4 \\
\hline \multicolumn{11}{|l|}{ Base line } \\
\hline & 2.2 & 2.5 & 23.7 & 3.9 & 7.2 & 47.6 & 12.3 & 0.7 & 0 & 0.1 \\
\hline \multicolumn{11}{|c|}{ After $2 \mathrm{wk}$ of the fat-free diet } \\
\hline & 1.0 & 2.9 & 21.4 & 5.7 & 5.2 & 49.7 & 12.8 & 0.9 & 0 & 0.3 \\
\hline
\end{tabular}

Eicosatrienoic acid was not present in measurable quantities in any fraction at base line. It appeared on the 1st day of the fat-free diet in the phospholipid and triglyceride fractions and increased in these two fractions to the end of Period A to values of 2.7 and $2.9 \%$, respectively. The eicosatrienoic acid of the cholesterol ester fraction did not appear until day 7 and reached only $0.8 \%$ by day 10 . Free fatty acid eicosatrienoic acid appeared at day 3 and reached only $0.4 \%$ by day 10 . Arachidonic acid did not change significantly.

During the 24-h fast, Period B, linoleic acid levels rose in all fractions. The greatest relative rise was a fourfold increase in free fatty acid linoleic acid from 1.8 to $7.6 \%$. Linoleic acid in the phospholipid and triglyceride fractions increased similarly from 2.8 to $10.3 \%$ and 2.9 to $10.3 \%$, respectively. Cholesterol ester linoleic acid increased also from 9.4 to $13.9 \%$.

The intermittent formula feeding of Period $\mathrm{C}$ resulted in a further increase in cholesterol ester linoleic acid, from 13.9 to $21.9 \%$. Phospholipid linoleic acid did not change. The linoleic acid of the free fatty acid and triglyceride fractions fell from 7.6 to $6.3 \%$ and 10.3 to $8.4 \%$, respectively.

Eicosatrienoic acid did not decrease during Period B, the $24-\mathrm{h}$ fast. Intermittent feeding produced no change in the cholesterol ester eicosatrienoic acid, slight decreases in free fatty acid and phospholipid eicosatrienoic acid, and a marked decrease in triglyceride eicosatrienoic acid from 3.5 to $1.3 \%$.

After administration of linoleic acid as $2.6 \%$ of calories in the repletion diet, Period D, the serum linoleic acid reached base-line levels in all fractions by day. 13. Eicosatrienoic acid had practically disappeared by day 13 being present as $0.3 \%$ of phospholipids and triglycerides and in a trace amount in cholesterol esters.

No clinical signs of EFA deficiency were detected in this subject.

Adipose tissue biopsies were done at the beginning and end of Study II (Table IV). The fatty acid composition was similar in the two samples which both contained linoleic acid as $12 \%$ of the total fatty acids.
Neither sample contained any detectable eicosatrienoic acid.

\section{DISCUSSION}

Two important conclusions emerged from these studies. First, EFA deficiency invariably occurred in healthy, well-nourished adult humans continuously fed a eucaloric fat-free diet made up of glucose and amino acids. This state of deficiency, as manifested by great decreases of linoleic acid in the plasma lipid classes and the appearance of eicosatrienoic acid, became detectable in 1 day and was fully manifested by 3 days. No real difference was noted between the intravenous and nasogastric routes of administration. Secondly, short periods of fasting or of intermittent feeding seemed to replete partially an EFA-deficient individual, presumably from EFA stores in the adipose tissues.

To be emphasized is that there were two requirements for the development of the EFA deficiency: first, a fat-free diet and, secondly, the continuous infusion of glucose which prevented the mobilization of the large stores of linoleate in the adipose tissues (12\% of the total fatty acids in our subject of Study II). A feast of glucose calories led to a famine of linoleate for the viscera and peripheral tissues despite the presence of 700 or more $\mathrm{g}$ of linoleate in the adipose tissues, enough to supply the EFA needs of the body for 233 days or more if it could be but mobilized.

Although linoleic acid decreased rapidly for the first 2 wk of the deficiency state, it did not decline further with time. In neither of these studies nor in those reported in the literature has linoleate ever disappeared completely from the serum. This may be due to the release of linoleate via tissue catabolism or the hydrolysis of biliary phospholipids, or perhaps to the substitution of isomers of linoleic acid, including C18:

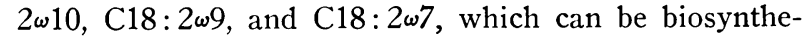
sized and which were not resolved by the gas chromatographic methods used. These isomers have been shown to occur in EFA-deficient rats (22).

Animal studies have shown, however, that when linoleic acid is not present as $1 \%$ or more of total calories 
5,8,11-eicosatrienoic acid appears $(2,8)$. This is the established biochemical indication of a metabolic deficiency state which has been repeatedly found characteristic of EFA deficiency $(2,8,12-15)$. Under normal circumstances, the body converts linoleic acid (which cannot be biosynthesized) to arachidonic acid. When linoleic acid is not present in adequate amounts, the same enzyme system elongates and desaturates oleic acid (C18:1), a fatty acid which can be biosynthesized by the body, and produces 5, 8, 11-eicosatrienoic acid rather than arachidonic acid. This latter reaction is competitively inhibited by linoleic acid (23).

The increases in linoleic acid during the periods of total fasting and the intermittent feeding in Study II and the decrease in eicosatrienoic acid during the intermittent feeding would seem to indicate that repletion was occurring. The source of this linoleic acid was presumably adipose tissue triglyceride which contained $12 \%$ linoleic acid and which was mobilized during the periods of fast. These results suggest a theoretical basis for a therapeutic measure to prevent EFA deficiency during the clinical situation of hyperalimentation feeding. Clearly, the fasting of malnourished patients would not be appropriate, but the incorporation of a fat source containing linoleate into the hyperalimentation diet, as suggested by MacFayden et al., might well prevent EFA deficiency (24). While they did not study the EFA status of their patients, these investigators infused a $10 \%$ soybean oil emulsion (Intralipid®, Cutter Laboratories, Inc., Berkeley, Calif.) along with a formula containing $25 \%$ dextrose, $4.2 \%$ amino acids, vitamins, and electrolytes for a period of $4 \mathrm{~h}$.

One point of interest which may be noted from examining the data during Periods A and B of our study is the source of the fatty acids in the free fatty acid fraction. It has been assumed that these are usually derived solely from adipose tissue. During Period A the relative fatty acid composition of the free fatty acid fraction was changing dramatically (Table III), yet the subject was not losing weight and presumably not mobilizing adipose tissue because of the continuous glucose infusion. Biopsies done at the beginning and end of the study showed similar linoleic acid contents of the adipose tissue, about $12 \%$ of the total fatty acids. In addition, the free fatty acid composition after the 24-h fast of Period B was similar to that at base line indicating that no gross changes in adipose tissue had occurred during Period A. The changes in triglyceride fatty acid composition during Period A were quite similar to those in the free fatty acid fraction. Since it is well established that the liver is the major site of triglyceride production, the free fatty acids might be derived via intravascular hydrolysis of triglycerides or, conceivably, via direct hepatic secretion of free fatty acids under the circumstances of this study with a shutdown of free fatty acid outflow from the adipose tissue depots.

Complete correction of EFA deficiency occurred in 2 wk in Study II with reinstitution of dietary linoleic acid at $2.6 \%$ of total calories. This is in agreement with the usual recommendation of $1-2 \%$ of total calories as linoleic acid, but somewhat less than Collins and coworkers' recommendation of $4 \%$ of calories (15). They found that when linoleic acid intake decreased from 6.4 to $2.2 \%$ of calories, phospholipid eicosatrienoic rose from 2.4 to $3.6 \%$. However, they were dealing with a severely depleted patient who, in addition, had short bowel syndrome and could not adequately absorb linoleic acid.

Collins et al. observed that arachidonic acid increased or decreased in direct response to dietary linoleic acid in their subject. Because of the biochemical pathway of linoleate to arachidonate, this seems logical. This correlation, however, was seen only in the phospholipid fraction during Study I in our studies. Perhaps similar changes would have developed in other fractions if the length of the dietary period was increased. The turnover time for arachidonic acid is somewhat slower than that for linoleic acid $(25,26)$.

In 1954 and 1958, Hanson, Wiese, and coworkers thoroughly investigated EFA deficiency in children (11, 27-30). They definitely established that a state of EFA deficiency can occur in growing humans. Their results were somewhat limited by their methodology which did not include gas-liquid chromatography, and results were expressed in terms of di-, tri-, and tetraenoic acid levels. Recently, in two studies, EFA deficiency occurred in infants receiving fat-free hyperalimentation diets similar to the ones used in the studies reported here $(12,14)$.

Since the work of Dudrick, Wilmore, Varn, and Rhoads (31) in 1968, the use of hyperalimentation diets which include glucose and amino acids, but no fat, have been extensively used for the extended nourishment of severely ill patients. Collins et al. (15) have reported the occurrence of EFA deficiency in a patient with a bowel resection who was maintained by fat-free intravenous alimentation for 100 days. He had no clinical evidence of malnutrition except a dermatitis, but his serum phospholipids contained $10 \%$ 5,8,11-eicosatrienoic acid. A fat infusion containing $6.6 \%$ of total calories as linoleate was then administered daily for 12 days. This caused a sharp decline in the 5,8,11-eicosatrienoic acid from 9.9 to $1.2 \%$ of total phospholipid fatty acids.

Patients receiving fat-free parenteral hyperalimentation diets are often ill and nutritionally depleted. They seem unusually susceptible to infection (32). Perhaps one contributing cause of the infection might be the associated EFA deficiency $(3,8)$. In view of the studies

Essential Fatty Acid Deficiency in Man 
we have reported, it would now seem advisable to supplement parenteral calories with linoleic acid. The addition of small amounts of linoleic acid to the diet, perhaps as little at $1-2 \%$ of total calories, would avoid EFA deficiency.

\section{ACKNOWLEDGMENTS}

This paper was supported by research grants from the U. S. Public Health Service, National Heart and Lung Institute (HE-14,230), and from the General Clinical Research Centers Program (M01-FR-59) of the Division of Research Resources, National Institutes of Health.

\section{REFERENCES}

1. Burr, G. O., and M. D. Burr. 1929. A new deficiency disease produced by the rigid exclusion of fat from the diet. J. Biol. Chem. 82 : 345-367.

2. Holman, R. T. 1968. Essential fatty acid deficiency. Progress in the Chemistry of Fat and Other Lipids. Pergamon Press Inc., Elmsford, N. Y. 279-348.

3. Essential fatty acids, In The Vitamins. 1971. W. H. Sebrell, Jr. and R. S. Harris, editors. Academic Press, Inc., New York. 2nd edition. 3: 303-339.

4. White, A., P. Handler, and E. L. Smith. 1968. Principles of Biochemistry. McGraw-Hill Book Company, New York. 4th edition. 494-495.

5. Guarnieri, M., and R. M. Johnson. 1970. The essential fatty acids. Adv. Lipid Res. 8: 115-174.

6. Mohrhauer, H., and R. T. Holman. 1963. Alteration of the fatty acid composition of brain lipids by varying levels of dietary essential fatty acids. J. Neurochem. 10: 523-530.

7. Brown, W. R., A. E. Hansen, G. O. Burr, and I. McQuarrie. 1938. Effects of prolonged use of extremely low-fat diet on an adult human subject. J. Nutr. 16: 511-524.

8. Aaes-Jorgensen, E. 1961. Essential fatty acids. Physiol. Rev. 41: 1-51.

9. Soderhjelm, L., H. F. Wiese, and R. T. Holman. 1970. The role of polyunsaturated acids in human nutrition and metabolism. In Progress in the Chemistry of Fats and Other Lipids. R. T. Holman, editor. Pergamon Press, Inc., Elmsford, N. Y. 4: 555-585.

10. Hansen, A. E., and H. F. Wiese. 1954. Essential fatty acids and human nutrition. II. Serum level for unsaturated fatty acids in poorly-nourished infants and children. J. Nutr. 52 : 367-374.

11. Wiese, H. F., A. E. Hansen, and D. J. D. Adam. 1968. Essential fatty acids in infant nutrition. I. Linoleic acid requirements in terms of serum di-, tri,- and tetraenoic acid levels. J. Nutr. 66: 345-360.

12. Paulsrud, J. R., L. Pensler, C. F. Whitten, S. Stewart, and R. T. Holman. 1972. Essential fatty acid deficiency in adults induced by fat-free intravenous feeding. $\mathrm{Am}$. J. Clin. Nutr. 25 : 897-904.

13. Caldwell, M. D., H. T. Jonsson, and H. B. Othresen, Jr. 1972. Essential fatty acid deficiency in an infant receiving prolonged parenteral alimentation. J. Pediatr. 81 : 894-898.

14. White, H. B., M. D. Turner, M. S. Turner, and R. C. Miller. 1973. Blood lipid alterations in infants receiving intravenous fat-free alimentation. J. Pediatr. 83 : 305-313.

15. Collins, R. D., A. J. Sinclair, J. P. Royle, P. A. Coats, A. T. Maynard, and R. F. Leonard. 1971. Plasma lipids in human linoleic acid deficiency. Nutr. Mctab. 13: 150-167.

16. Sperry, W. M., and F. C. Brand. 1955. The determination of total lipids in blood serum. J. Biol. Chem. 213: 69-76.

17. Mangold, H. K. 1961. Thin-layer chromatography of lipids. J. Am. Oil Chem. Soc. 38: 708-727.

18. Morrison, W. R., and L. M. Smith. 1964. Preparation of fatty acid methyl esters and dimethylacetates from lipids with boron fluoride-methanol. J. Lipid Res. 5: 600-608.

19. Huntsberger, D. V., and P. E. Leaverton. 1970. Statistical Inference in the Biomedical Sciences. Allyn and Bacon, Inc., Boston. 135-136.

20. Nickaman, M. Z., C. C. Swelley, and R. E. Olson. 1969. Plasma fatty dcids in normolipemic and hyperlipemic subjects during fasting and after linoleate feeding. $\mathrm{Am}$. J. Clin. Nutr. 22 : 1070-1083.

21. Antonis, A., and I. Bersohn. 1962. The influence of diet serum lipids in South African white and Bantu prisoners. Am. J. Clin. Nutr. 10: 484-499.

22. Sand, D., N. Sen, and H. Schlenk. 1965. Positional isomerism of unsaturated fatty acids in the rat and quantification of isomeric mixtures. J. Am. Oil Chem. Soc. 42: 511-516.

23. Mead, J. F. 1968. The metabolism of the polyunsaturated fatty acids. In Progress in the Chemistry of Fats and Other Lipids. R. T. Holman, editor. Pergamon Press, Inc., Elmsford, N. Y. 9: 161-192.

24. MacFayden, B. V., S. J. Dudrick, E. P. Tagudar, A T. Maynard, D. K. Law, and J. E. Rhoads. 1973. Triglyceride and free fatty acid clearances in patients receiving complete parenteral nutrition using a ten percent soybean oil emulsion. Surg. Gynecol. Obst. 137 : 813-815.

25. Brenner, R. R., and A. M. Nervi. 1965. Kinetics of linoleic and arachidonic acid incorportion and eicosatrienoic depletion in the lipids of fat-deficient rats fed methyl linoleate and arachidonate. J. Lipid Res. 6: 363-368.

26. Coots, R. H. 1965. Metabolism of arachidonic acid-1-1 ${ }^{14} \mathrm{C}$ in the rat. J. Lipid Res. $6:$ 494-497.

27. Wiese, H. F., R. H. Gibbs, and A. E. Hansen. 1954 Essential fatty acids and human nutrition. I Serum level for unsaturated fatty acids in healthy children. J. Nutr. 52: 355-365.

28. Hansen, A. E., and H. F. Wiese. 1954. Essential fatty acids and human nutrition. II. Serum levels for unsaturated fatty acids in poorly-nourished infants and children. J. Nutr. 52: 367-374.

29. Adam, D. J. D., A. E. Hansen, and H. F. Wiese. 1958. Essential fatty acids in infant nutrition. II. Effect of linoleic acid on caloric intake. J. Nutr. 86: 555-564.

30. Hansen, A. E., M. E. Haggard, A. N. Boelsche, D. J. D. Adam, and H. F. Wiese. 1958. Essential fatty acids in infant nutrition. III. Clinical manifestations of linoleic acid deficiency. J. Nutr. 66: 565-576.

31. Dudrick, S. J., D. W. Wilmore, H. M. Vars, and J. E. Rhoads. 1968. Long-term total parenteral nutrition with growth, development, and positive nitrogen balance. Surgery (St. Louis). 64: 134-142.

32. Craddock, P. R., Y. Yawata, L. VanSanten, S. Gilberstadt, S. Silvis, and H. S. Jacob. 1974. Acquired phagocytic dysfunction: a complication of the hypophosphatemia of parenteral hyperalimentation. $N$. Engl. J. Med. 200: 1403-1407. 